Article

\title{
Threshold-Effect Association of Dietary Cholesterol Intake with Dyslipidemia in Chinese Adults: Results from the China Health and Nutrition Survey in 2015
}

\author{
Qiumin Huang, Hongru Jiang, Bing Zhang, Huijun Wang, Xiaofang Jia, Feifei Huang, \\ Liusen Wang and Zhihong Wang *
}

National Institute for Nutrition and Health, Chinese Center for Disease Control and Prevention, 29 Nanwei Road, Beijing 100050, China; qmhuangx@163.com (Q.H.); jhr_seven@163.com (H.J.);

zzhangb327@aliyun.com (B.Z.); wanghj@ninh.chinacdc.cn (H.W.); jiaxf@ninh.chinacdc.cn (X.J.);

fayekobe@163.com (F.H.); wangliusen1993@163.com (L.W.)

* Correspondence: wangzh@ninh.chinacdc.cn; Tel.: +86-10-6623-7076

Received: 9 November 2019; Accepted: 26 November 2019; Published: 27 November 2019

check for updates

\begin{abstract}
The association of dietary cholesterol intake with dyslipidemia and subtypes is controversial. This study aimed to examine the association of dietary cholesterol intake with dyslipidemia and subtypes in Chinese adults. Using data from the China Health and Nutrition Survey (CHNS) in 2015, the present study selected 4383 participants aged 18-59 years who were free of diabetes, apoplexy, and myocardial infarction disease. Information was obtained on dietary intake, anthropometric measurements, and blood laboratory measurements. Dietary cholesterol intake was calculated based on the data collected by consecutive 3 days $24 \mathrm{~h}$ recalls combined with the weighing of household seasonings and categorized by 11 levels: The first 10 levels in ranges of $50 \mathrm{mg} /$ day and the 11th level at $\geq 500 \mathrm{mg}$ /day. Dyslipidemia, hypercholesterolemia, hypertriglyceridemia, low-density lipoprotein (LDL)-hypercholesterolemia, and high-density lipoprotein (HDL)-hypocholesterolemia were defined based on the Chinese adult dyslipidemia prevention guide (2016 edition). Multivariable logistic regressions were performed to examine the association of dietary cholesterol intake levels with dyslipidemia and subtypes. The prevalence of dyslipidemia was $37.5 \%$ among Chinese adults in 2015 (hypercholesterolemia 9.6\%, HDL-hypocholesterolemia 21.1\%, LDL-hypercholesterolemia $12.7 \%$, and hypertriglyceridemia $15.2 \%$ ). The lowest prevalence of hypercholesterolemia and LDL-hypercholesterolemia was $6.7 \%$ and $9.4 \%$, respectively, which was relative to a dietary cholesterol intake level of 100.0 to $<150.0 \mathrm{mg} /$ day. After adjusting for all potential confounders, adults with the highest dietary cholesterol intake level of $\geq 500 \mathrm{mg} /$ day compared with the dietary cholesterol intake of 100.0 to $<150.0 \mathrm{mg} /$ day showed one-time higher odds of hypercholesterolemia (odds ratios (OR) 2.0, 95\% confidence intervals (CI) 1.3-3.3), as well as LDL-hypercholesterolemia (OR 2.0, 95\% CI 1.3-3.0), but a null association of dietary cholesterol intake with dyslipidemia, hypertriglyceridemia, and HDL-hypocholesterolemia. The study suggested that a dietary cholesterol intake level of $500 \mathrm{mg} /$ day and above may be a threshold point for high odds of hypercholesterolemia and LDL-hypercholesterolemia.
\end{abstract}

Keywords: dietary cholesterol; dyslipidemia; hypercholesterolemia; LDL-hypercholesterolemia; HDL-hypocholesterolemia; hypertriglyceridemia; Chinese adults

\section{Introduction}

Dyslipidemia is abnormal lipid metabolism characterized by any one of hypercholesterolemia, high-density lipoprotein (HDL)-hypocholesterolemia, low-density lipoprotein 
(LDL)-hypercholesterolemia, and hypertriglyceridemia [1]. Many studies suggested the key role of controlling dyslipidemia in the prevention of cardiovascular disease [2-4], the leading cause of mortality in China [5]. It is, therefore, crucial to identify modifiable risk factors, like dietary intake, to control the epidemic of dyslipidemia and related disease burden in China.

The association of dietary cholesterol intake with dyslipidemia has been gaining a high level of concern due to inconsistent evidence across studies. In 2017, Mente et al. reported that dietary cholesterol intake was associated with an increase in both serum total cholesterol (TC) and LDL-C and also with the increased LDL-C to HDL-C ratio [6]. A meta-analysis study of 17 randomized control trial studies conducted from 1974 to 1999 revealed that the addition of $100 \mathrm{mg}$ of dietary cholesterol increased the total/HDL cholesterol ratio [7]. And results from the controlled feeding studies showed changes in serum cholesterol ranging from 2.2 to $4.5 \mathrm{mg} / \mathrm{dL}$ per $100 \mathrm{mg} /$ day of change in dietary cholesterol [8]. On the contrary, others have shown a null association [9,10], because investigators have reported that increased intake of dietary cholesterol (exogenous) was associated with the decreased synthesis of endogenous de novo cholesterol, possibly as a compensatory mechanism that keeps cholesterol homeostasis constant [11,12]. In China, one study using China Health and Nutrition Survey (CHNS) 2009 found that the adults with a high intake of dietary cholesterol ( $\geq 300.0 \mathrm{mg} / \mathrm{day})$ had higher odds of hypercholesterolemia than the adults with intake of $<300 \mathrm{mg} /$ day [13]. Another study using the Shanghai diet and health survey (SDHS) showed $60 \%$ higher odds of dyslipidemia prevalence among Shanghai adults with a top quintile of dietary cholesterol intake $(\geq 538.0 \mathrm{mg} /$ day $)$ as compared to the odds with a bottom quintile ( $<193.1 \mathrm{mg} /$ day) [14].

Although both Chinese dietary guidelines and the dietary guidelines for Americans eliminated the recommendation of no more than $300.0 \mathrm{mg} /$ day of dietary cholesterol intake [15,16]. One study showed that Chinese adults in higher quantile had a much bigger increase in dietary cholesterol intake from 1991 to 2011 as compared with those in lower quantile. The current evidence does not seem enough to avoid any recommendation of dietary cholesterol intake, given the controversial results over studies [17]. To better understand their relationship, this study aimed to fully examine the dose-response association of dietary cholesterol intake, by refining its grouping, with prevalence of dyslipidemia and subtypes among Chinese adults aged 18-59 years using the data collected in CHNS 2015.

\section{Subjects and Methods}

\subsection{Study Population}

Data for this analysis were from the CHNS, established by the National Institute for Nutrition and Health, Chinese Center for Disease Control and Prevention, and the University of North Carolina at Chapel Hill. The CHNS is an ongoing series of longitudinal household surveys investigating how social and economic changes in China affect various nutrition- and health-related outcomes. The CHNS was conducted in 9 rounds across 9 provinces (autonomous regions) during the period of 1989-2009. The 9th round was conducted in 2011 across 12 provinces (autonomous regions) with the additions of Beijing, Shanghai, and Chongqing, and the 10th round was conducted in 2015 across 15 provinces (autonomous regions) with the additions of Shanxi, Yunnan, and Zhejiang. Multistage, random cluster sampling was used to select the survey sample in each province to ensure that urban and rural areas were represented in the CHNS. The survey design and methods have been described in detail elsewhere [18].

Our analysis used the 10th-round survey of CHNS in 2015. From all subjects aged 18-59 years who had complete socioeconomic status and demographic data, 3 days $24 \mathrm{~h}$ dietary recall data, and biochemical data, excluding those with diabetes, apoplexy, and myocardial infarction disease, those with implausible energy intakes ( $<800 \mathrm{kcal} /$ day or $>6000 \mathrm{kcal} /$ day for men and $<600 \mathrm{kcal} /$ day or $>4000 \mathrm{kcal} / \mathrm{day}$ for women), and those with missing dietary cholesterol intake and serum lipids. Subjects with diabetes, stroke, and myocardial infarction disease were defined as having hospital 
records diagnosed by professional doctors or receiving treatment for these diseases. A total of 4383 adults (1949 males and 2434 females) were included in the final analyses. This study was reviewed and approved by the institutional review board of the University of North Carolina at Chapel Hill and the Chinese Center for Disease Control and Prevention. Written informed consent was obtained from all subjects.

\subsection{Assessment of Dietary Cholesterol Intake}

CHNS 2015 collected dietary data using 3 consecutive $24 \mathrm{~h}$ recalls ( 2 weekdays and 1 weekend day) for each individual and weighed seasonings in the household inventory over the same period. Trained health workers interviewed the participants on each of those days to collect all food consumption (type, amounts, type of meal, and place of consumption) at home and away from home during the preceding $24 \mathrm{~h}$. Individuals' consumption of seasonings was calculated according to eating times at home and individuals' energy proportion among family members. Details of the collection method are described elsewhere $[18,19]$. The individual daily intake of dietary energy, macronutrients, cholesterol, and micronutrients was calculated according to daily food and condiments consumption using the China Food Composition Table [19]. Dietary cholesterol intake levels were categorized as $<50 \mathrm{mg} / \mathrm{day}$, 50 to $<100 \mathrm{mg} /$ day, 100 to $<150 \mathrm{mg} /$ day, 150 to $<200 \mathrm{mg} /$ day, 200 to $<250 \mathrm{mg} /$ day, 250 to $<300 \mathrm{mg} /$ day, 300 to $<350 \mathrm{mg} /$ day, 350 to $<400 \mathrm{mg} /$ day, 400 to $<450 \mathrm{mg} /$ day, 450 to $<500 \mathrm{mg} /$ day, and $\geq 500 \mathrm{mg} / \mathrm{day}$.

\subsection{Definitions of Dyslipidemia}

For lipids detection, a fasting blood sample was collected by experienced physicians, phlebotomists, or nurses via venipuncture. Plasma and serum samples were frozen and stored at $-80{ }^{\circ} \mathrm{C}$ for later laboratory analysis. Blood samples were analyzed at the laboratory of the National Institute for Nutrition and Health. Serum concentrations of triglycerides (TG) and total cholesterol (TC) were measured using the glycerol-phosphate oxidase method, and low-density lipoprotein cholesterol (LDL-C) and high-density lipoprotein cholesterol (HDL-C) were measured using the polyethylene glycol-modified enzyme method by determiner regents (Kyowa Medex Co. Ltd., Tokyo, Japan) on a Hitachi 7600 automated analyzer (Hitachi Inc., Tokyo, Japan).

According to the Chinese adult dyslipidemia prevention guide (2016 edition), serum TC concentrations greater than $6.2 \mathrm{mmol} / \mathrm{L}(240 \mathrm{mg} / \mathrm{dL})$ were considered to be hypercholesterolemia, serum TG concentrations greater than $2.3 \mathrm{mmol} / \mathrm{L}(200 \mathrm{mg} / \mathrm{dL})$ were considered to be hypertriglyceridemia, serum LDL-C concentrations greater than $4.1 \mathrm{mmol} / \mathrm{L}(160 \mathrm{mg} / \mathrm{dL})$ were considered to be LDL-hypercholesterolemia, and serum HDL-C concentrations less than $1.0 \mathrm{mmol} / \mathrm{L}(40 \mathrm{mg} / \mathrm{dL})$ were considered to be HDL-hypocholesterolemia [20]. Dyslipidemia was defined as having at least one of the following: Hypercholesterolemia, hypertriglyceridemia, LDL-hypercholesterolemia, or HDL-hypocholesterolemia.

\subsection{Assessment of Covariates}

Information on sociodemographic (e.g., gender, age, geographic region, education, and income) and lifestyle (e.g., smoking and drinking status and physical activity) variables were collected through a combination of self-administered and interviewer-administered questionnaires. All the involved provinces were grouped into 3 major economic zones (central, east, and west) based on China's "Seventh Five-Year Plan". The west region included the city of Chongqing and the provinces of Guizhou, Yunnan, Shanxi, and Guangxi. The central region included the provinces of Heilongjiang, Henan, Hubei, and Hunan. The east region included the cities of Beijing and Shanghai and the provinces of Jiangsu, Zhejiang, Shandong, and Shanxi. Education was categorized as primary school or less, secondary school, and high school or more. The yearly income per capita was obtained by asking the head of the household and categorized into tertiles (low, medium, and high). Smoking and drinking statuses were categorized as current and current non-. Physical activity was assessed by metabolic equivalent-hours/week (MET-h/week), which equaled intensity of physical activity multiplied by 
physical activity time. The intensity of physical activity assignments referred to the American College of Sports Medicine Association's recommended standard [21].

Weight and height were measured to the nearest $0.1 \mathrm{~kg}$ and $0.1 \mathrm{~cm}$, respectively, with the participants in lightweight clothing and without shoes. Body mass index (BMI) was calculated from measured weight $(\mathrm{kg})$ and height $(\mathrm{m})$ squared $\left(\mathrm{kg} / \mathrm{m}^{2}\right)$ [22]. Three blood pressure (BP) measurements were taken using regularly calibrated mercury sphygmomanometers on the right arm with the participant in a seated position after at least 5 minutes of rest in a quiet room. Participants were advised to avoid cigarette smoking, alcohol, caffeinated beverages, and exercise for at least 30 minutes before the measurement. Systolic blood pressure (SBP) was measured at the first appearance of a pulse sound (Korotkoff Phase 1) and diastolic blood pressure (DBP) at the disappearance of the pulse sound (Korotkoff Phase 5). Hypertension was defined as having SBP $\geq 140 \mathrm{~mm} \mathrm{Hg}$ and/or DBP $\geq 90 \mathrm{~mm} \mathrm{Hg}$ according to the mean of three BP measurements or using antihypertensive medications.

\subsection{Statistical Analysis}

Firstly, statistical interaction tests between dietary cholesterol intake and gender, age, and BMI were performed, and no statistical significance was found. The median value for continuous variables was presented, given the non-normal distribution tested by Kolmogorov-Smirnov and percentage of the total for categorical variables. Differences among daily dietary cholesterol intake levels were examined using the Wilcoxon rank-sum and Kruskal-Wallis $\mathrm{H}$ test for continuous variables and Chi-square test for categorical variables. A Cochran-Armitage test for trend was conducted to test the trend for prevalence of dyslipidemia, hypercholesterolemia, hypertriglyceridemia, HDL-hypocholesterolemia, and LDL-hypercholesterolemia with the increase of dietary cholesterol intake.

A series of multivariate binomial logistic regression models were conducted to assess the odds ratios (OR) and $95 \%$ confidence intervals (CI) by levels of dietary cholesterol intake, adjusting for potential confounders, including demographics, socioeconomic status, lifestyle, related intake of food groups and nutrients, and BMI value, in turn considering no multicollinearity. In addition, the linear trends were tested by assigning median values to levels of dietary cholesterol intake, and the variable modeled as a continuous term.

All statistical analyses were performed using SAS version 9.4 software (SAS Institute Inc., Cary, NC, USA). Formal hypothesis testing was two-sided with a significance level of 0.05 .

\section{Results}

\subsection{Baseline Characteristics}

The baseline characteristics of participants across levels of dietary cholesterol intake are summarized in Table 1. Of all participants, the daily dietary cholesterol intakes for gender, age group, geographic region, educational level, yearly income, drinking status, hypertension history, and dietary nutrient intakes were statistically significant $(p<0.05)$. Participants with the highest daily dietary cholesterol level consumed more dietary intakes of energy, carbohydrate, protein, fat, fiber, vitamin $C$, vitamin $\mathrm{E}$, niacin, and selenium $(p<0.001)$. 
Table 1. Basic characteristics of the study population ${ }^{1}$.

\begin{tabular}{|c|c|c|c|c|c|c|c|c|c|c|c|c|c|}
\hline Characteristics & Total & $\begin{array}{c}<50.0 \\
\mathrm{mg} / \mathrm{day}\end{array}$ & $\begin{array}{c}50.0-<100.0 \\
\text { mg/day }\end{array}$ & $\begin{array}{c}100.0-<150.0 \\
\mathrm{mg} / \mathrm{day}\end{array}$ & $\begin{array}{c}150.0-<200.0 \\
\mathrm{mg} / \mathrm{day}\end{array}$ & $\begin{array}{c}200.0-<250.0 \\
\text { mg/day }\end{array}$ & $\begin{array}{c}250.0-<300.0 \\
\mathrm{mg} / \mathrm{day}\end{array}$ & $\begin{array}{c}300.0-<350.0 \\
\text { mg/day }\end{array}$ & $\begin{array}{c}350.0-<400.0 \\
\mathrm{mg} / \mathrm{day}\end{array}$ & $\begin{array}{c}400.0-<450.0 \\
\text { mg/day }\end{array}$ & $\begin{array}{c}450.0-<500.0 \\
\mathrm{mg} / \mathrm{day}\end{array}$ & $\begin{array}{l}\geq 500.0 \\
\mathrm{mg} / \text { day }\end{array}$ & $p$-value \\
\hline Percent of all adults (\%) & 100.0 & 10.0 & 9.5 & 11.7 & 11.2 & 11.1 & 9.7 & 8.3 & 7.5 & 5.1 & 3.4 & 12.6 & \\
\hline Gender (\%) & & & & & & & & & & & & & $<0.001$ \\
\hline Male & 44.5 & 37.8 & 39.9 & 42.5 & 41.2 & 41.6 & 44.7 & 44.8 & 50.0 & 49.8 & 52.7 & 52.4 & \\
\hline Female & 55.5 & 62.2 & 60.1 & 57.5 & 58.8 & 58.4 & 55.3 & 55.2 & 50.0 & 50.2 & 47.3 & 47.6 & \\
\hline Age (\%) & & & & & & & & & & & & & $<0.001$ \\
\hline $18-44$ years & 41.4 & 31.2 & 43.5 & 40.3 & 44.9 & 38.9 & 47.3 & 46.4 & 42.4 & 39.5 & 38.7 & 41.3 & \\
\hline 45-59 years & 58.6 & 68.8 & 56.5 & 59.7 & 55.1 & 61.1 & 52.7 & 53.6 & 57.6 & 60.5 & 61.3 & 58.7 & \\
\hline Geographic region & & & & & & & & & & & & & $<0.001$ \\
\hline Central & 35.9 & 43.6 & 35.6 & 35.6 & 40.4 & 35.6 & 33.9 & 31.3 & 33.0 & 39.5 & 36.0 & 31.2 & \\
\hline East & 38.5 & 40.8 & 35.6 & 36.0 & 28.4 & 33.3 & 37.4 & 41.5 & 47.3 & 39.5 & 41.3 & 46.9 & \\
\hline West & 25.7 & 15.6 & 28.9 & 28.4 & 31.2 & 31.1 & 28.7 & 27.2 & 19.7 & 21.1 & 22.7 & 21.9 & \\
\hline \multicolumn{14}{|l|}{ Educational level } \\
\hline$\leq$ Primary school & 21.2 & 35.1 & 19.7 & 24.3 & 21.8 & 21.8 & 20.9 & 16.8 & 16.4 & 16.6 & 14.7 & 17.0 & $<0.001$ \\
\hline Secondary school & 38.0 & 39.9 & 44.7 & 38.8 & 37.4 & 36.2 & 32.9 & 39.0 & 35.8 & 39.5 & 35.3 & 37.5 & \\
\hline$\geq$ High school & 40.8 & 25.0 & 35.6 & 37.0 & 40.8 & 42.0 & 46.1 & 44.2 & 47.9 & 44.0 & 50.0 & 45.5 & \\
\hline Yearly Income & & & & & & & & & & & & & $<0.001$ \\
\hline Low & 33.4 & 45.2 & 38.5 & 36.4 & 32.2 & 32.5 & 33.2 & 30.5 & 26.4 & 28.7 & 30.0 & 27.9 & \\
\hline Medium & 33.3 & 31.2 & 35.6 & 32.3 & 33.3 & 36.0 & 32.5 & 30.8 & 38.2 & 36.3 & 32.7 & 30.4 & \\
\hline High & 33.3 & 23.6 & 26.0 & 31.3 & 34.5 & 31.5 & 34.4 & 38.7 & 35.5 & 35.0 & 37.3 & 41.7 & \\
\hline BMI $\left(\mathrm{kg} / \mathrm{m}^{2}\right)$ & 24.3 & 24.2 & 24.0 & 23.9 & 23.7 & 24.0 & 23.9 & 24.0 & 24.6 & 24.0 & 23.6 & 24.4 & 0.051 \\
\hline Smoking (\%) & 24.0 & 22.3 & 20.9 & 22.7 & 24.9 & 24.1 & 23.1 & 23.9 & 23.9 & 28.7 & 23.3 & 28.1 & 0.319 \\
\hline $\begin{array}{l}\text { Dmoking ( }(\%) \\
\text { Drinking }(\%)\end{array}$ & 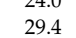 & 24.8 & 26.2 & 29.2 & $\begin{array}{l}28.9 \\
28.6\end{array}$ & $\begin{array}{l}2.1 \\
29.2\end{array}$ & 28.9 & $\begin{array}{l}23.9 \\
27.8\end{array}$ & 29.7 & 32.7 & $\begin{array}{l}2.3 \\
35.3\end{array}$ & $\begin{array}{l}28.1 \\
35.1\end{array}$ & $\begin{array}{l}0.319 \\
0.032\end{array}$ \\
\hline Hypertension (\%) & 26.3 & 33.7 & 21.6 & 25.2 & 25.1 & 24.5 & 27.1 & 22.5 & 26.4 & 29.6 & 24.7 & 28.8 & 0.006 \\
\hline Physical activity (MET-h/week) & 30.3 & 32.5 & 29.0 & 20.0 & 21.6 & 37.8 & 32.0 & 42.8 & 30.6 & 26.4 & 28.3 & 35.5 & 0.253 \\
\hline \multicolumn{14}{|l|}{ Dietary intakes } \\
\hline Energy (kcal/day) & 1869.7 & 1650.5 & 1610.9 & 1723.4 & 1761.2 & 1781.5 & 1886.9 & 1922.6 & 2029.9 & 2085.8 & 2011.9 & 2286.0 & $<0.001$ \\
\hline Carbohydrate (g/day) & 230.4 & 239.7 & 211.3 & 224.2 & 218.3 & 218.8 & 229.5 & 228.1 & 244.8 & 237.1 & 244.0 & 253.3 & $<0.001$ \\
\hline $\begin{array}{l}\text { Fat (g/day) } \\
\text { (g/ual }\end{array}$ & 70.7 & 46.7 & 58.7 & 65.1 & 68.1 & 74.8 & 69.1 & 76.3 & 78.1 & 82.3 & 76.3 & 91.8 & $<0.001$ \\
\hline Protein (g/day) & 58.4 & 44.6 & 46.9 & 50.9 & 53.0 & 55.6 & 61.2 & 63.2 & 68.6 & 68.0 & 71.8 & 84.2 & $<0.001$ \\
\hline Fiber (g/day) & $\begin{array}{l}50.4 \\
9.9\end{array}$ & $\begin{array}{l}44.0 \\
10.0\end{array}$ & $\begin{array}{l}\begin{array}{l}40.9 \\
9.4\end{array} \\
\end{array}$ & $\begin{array}{l}50.9 \\
9.2\end{array}$ & $\begin{array}{l}5.0 \\
9.1\end{array}$ & $\begin{array}{l}30.0 \\
9.6\end{array}$ & $\begin{array}{l}01.2 \\
10.0\end{array}$ & $\begin{array}{l}\text { D. } 2.2 \\
10.1\end{array}$ & $\begin{array}{l}\text { D.o. } \\
10.3\end{array}$ & $\begin{array}{l}0.0 \\
10.2\end{array}$ & 10.5 & $\begin{array}{l}\text { Of.2 } \\
11.6\end{array}$ & $<0.001$ \\
\hline Vitamin C (mg/day) & 61.8 & 52.5 & 55.0 & 56.7 & 54.5 & 61.0 & 60.0 & 67.7 & 64.7 & 72.7 & 69.2 & 76.6 & $<0.001$ \\
\hline Vitamin E (mg/day) & 24.5 & 22.6 & 22.0 & 24.9 & 23.9 & 22.9 & 22.9 & 24.3 & 25.7 & 26.2 & 26.4 & 28.8 & $<0.001$ \\
\hline Niacin (mg/day) & 13.4 & 10.0 & 11.2 & 11.9 & 12.4 & 13.2 & 14.0 & 14.7 & 14.7 & 15.0 & $\begin{array}{l}2.4 \\
16.2\end{array}$ & $\begin{array}{l}2.0 \\
17.8\end{array}$ & $<0.001$ \\
\hline Selenium ( $\mu \mathrm{g} /$ day $)$ & 39.2 & 30.3 & 30.2 & 32.7 & 34.2 & 37.2 & 39.8 & 42.0 & 48.4 & 49.0 & 47.6 & 58.3 & $<0.001$ \\
\hline
\end{tabular}

Abbreviation: BMI = body mass index; TC = total cholesterol; HDL-C = high-density lipoprotein cholesterol; LDL-C = low-density lipoprotein cholesterol; TG = triglyceride. ${ }^{1}$ : Data of gender, age, geographic region, educational level, yearly income, smoking, drinking, and hypertension are expressed as $n$ (\%); data of BMI, energy, dietary carbohydrate, dietary fat, dietary protein, dietary fiber, dietary vitamin C, dietary vitamin E, dietary niacin, dietary selenium, TC, TG, HDL-C, and LDL-C are expressed as median. 
3.2. Prevalence of Dyslipidemia Dyslipidemia, Hypercholesterolemia, Hypertriglyceridemia, HDLHypocholesterolemia, and LDL-Hypercholesterolemia in Participants

Of all participants, 37.5\% had dyslipidemia, 9.6\% had hypercholesterolemia, 15.2\% had hypertriglyceridemia, 21.1\% had HDL-hypocholesterolemia, and 12.7\% had LDL-hypercholesterolemia. The prevalence of dyslipidemia, hypercholesterolemia, and LDL-hypercholesterolemia was significant for different dietary cholesterol intake levels and showed an upward trend with the increase of dietary cholesterol intake levels $(p<0.05)$. However, the lowest prevalence of hypercholesterolemia and LDL-hypercholesterolemia was $6.7 \%$ and $9.4 \%$, respectively, which was relative to a cholesterol intake of 100.0-150.0 mg/day. In contrast, the prevalence and trend of dyslipidemia, hypertriglyceridemia, and HDL-hypocholesterolemia did not differ among different dietary cholesterol intake levels (see Table 2).

3.3. Associations of Dietary Cholesterol Intake Levels with Dyslipidemia, Hypercholesterolemia, Hypertriglyceridemia, HDL-Hypocholesterolemia, and LDL-Hypercholesterolemia

Table 3 shows the ORs of dyslipidemia, hypercholesterolemia, hypertriglyceridemia, HDL-hypocholesterolemia, and LDL-hypercholesterolemia according to levels of dietary cholesterol intake in Chinese adults. After adjusting for potential confounders in each model, compared with the dietary cholesterol intake of 100.0 to $<150.0 \mathrm{mg} /$ day, adults consuming the dietary cholesterol intake of 50.0 to $<100.0 \mathrm{mg} /$ day were found to decrease their odds of hypercholesterolemia, and adults consuming the dietary cholesterol intake of 200.0 to $<250.0 \mathrm{mg} /$ day, of 250.0 to $<300.0 \mathrm{mg} / \mathrm{day}$, and of $\geq 500.0 \mathrm{mg} / \mathrm{day}$ showed significantly higher odds of hypercholesterolemia and LDL-hypercholesterolemia.

After adjusting for all potential confounders, adults with the highest intake of dietary cholesterol showed two times the odds of hypercholesterolemia (OR 2.0, 95\% CI 1.3-3.3), as well as LDL-hypercholesterolemia (OR 2.0, 95\% CI 1.3-3.0). A null association of dietary cholesterol intake was observed, with odds of hypertriglyceridemia and HDL-hypocholesterolemia. None of the linear trend tests were statistically significant between dietary cholesterol intake level and the odds of dyslipidemia, hypercholesterolemia, hypertriglyceridemia, HDL-hypocholesterolemia, and LDL-hypercholesterolemia in adults. 
Table 2. Prevalence of dyslipidemia and its subtypes (\%).

\begin{tabular}{|c|c|c|c|c|c|c|c|c|c|c|c|c|c|}
\hline Variables & Total & $\begin{array}{l}<50.0 \\
\mathrm{mg} / \text { day }\end{array}$ & $\begin{array}{c}50.0-<100.0 \\
\mathrm{mg} / \text { day }\end{array}$ & $\begin{array}{c}100.0-<150.0 \\
\mathrm{mg} / \text { day }\end{array}$ & $\begin{array}{c}150.0-<200.0 \\
\mathrm{mg} / \text { day }\end{array}$ & $\begin{array}{c}200.0-<250.0 \\
\text { mg/day }\end{array}$ & $\begin{array}{c}250.0-<300.0 \\
\mathrm{mg} / \text { day }\end{array}$ & $\begin{array}{c}300.0-<350.0 \\
\mathrm{mg} / \text { day }\end{array}$ & $\begin{array}{c}350.0-<400.0 \\
\mathrm{mg} / \text { day }\end{array}$ & $\begin{array}{c}400.0-<450.0 \\
\mathrm{mg} / \text { day }\end{array}$ & $\begin{array}{c}450.0-<500.0 \\
\mathrm{mg} / \text { day }\end{array}$ & $\begin{array}{l}\geq 500.0 \\
\mathrm{mg} / \text { day }\end{array}$ & $p$-trend $*$ \\
\hline Dyslipidemia *, 市 & 37.5 & 33.3 & 36.8 & 36.4 & 35.7 & 41.2 & 35.8 & 37.4 & 39.7 & 35.9 & 38.0 & 41.1 & 0.025 \\
\hline Hypercholesterolem & 9.6 & 8.0 & 9.6 & 6.7 & 8.8 & 11.1 & 11.3 & 8.5 & 7.3 & 9.0 & 11.3 & 13.6 & 0.004 \\
\hline Hypertriglyceridemia & 15.2 & 13.3 & 16.8 & 14.1 & 16.1 & 18.7 & 13.2 & 14.8 & 14.2 & 11.7 & 10.0 & 17.8 & 0.863 \\
\hline HDL-hypocholesterolemia & 21.1 & 19.5 & 22.4 & 23.7 & 20.0 & 22.4 & 18.4 & 22.8 & 22.1 & 19.7 & 20.7 & 20.1 & 0.584 \\
\hline LDL-hypercholesterolemia *, & 12.7 & 9.6 & 10.1 & 9.4 & 11.8 & 14.6 & 13.4 & 12.4 & 13.0 & 13.9 & 11.3 & 18.3 & $<0.001$ \\
\hline
\end{tabular}

* Showed a significant difference in the prevalence of dyslipidemia and its four subtypes tested by Chi-square test $(p<0.05)$. $\ddagger$ Showed an uptrend tested by the Cochran-Armitage test for trend $(p<0.05)$.

Table 3. Adjusted ORs (95\% CI) for four types of dyslipidemia across the levels of dietary cholesterol intake in adults.

\begin{tabular}{|c|c|c|c|c|c|c|c|c|c|c|c|c|}
\hline Variables & $\begin{array}{l}<50.0 \\
\text { mg/day }\end{array}$ & $\begin{array}{c}50.0-<100.0 \\
\mathrm{mg} / \mathrm{day}\end{array}$ & $\begin{array}{c}100.0-<150.0 \\
\mathrm{mg} / \mathrm{day}\end{array}$ & $\begin{array}{c}150.0-<200.0 \\
\mathrm{mg} / \mathrm{day}\end{array}$ & $\begin{array}{c}200.0-<250.0 \\
\mathrm{mg} / \mathrm{day}\end{array}$ & $\begin{array}{c}250.0-<300.0 \\
\mathrm{mg} / \mathrm{day}\end{array}$ & $\begin{array}{c}300.0-<350.0 \\
\text { mg/day }\end{array}$ & $\begin{array}{c}350.0-<400.0 \\
\text { mg/day }\end{array}$ & $\begin{array}{c}400.0-<450.0 \\
\mathrm{mg} / \mathrm{day}\end{array}$ & $\begin{array}{c}450.0-<500.0 \\
\mathrm{mg} / \text { day }\end{array}$ & $\begin{array}{l}\geq 500.0 \\
\mathrm{mg} / \text { day }\end{array}$ & $p$-Trend ${ }^{1}$ \\
\hline \multicolumn{13}{|c|}{ Dyslipidemia } \\
\hline Model $1^{2}$ & $0.9(0.7,1.2)$ & $1.0(0.8,1.4)$ & Reference & $1.0(0.8,1.3)$ & $1.2(0.9,1.6)$ & $1.0(0.7,1.3)$ & $1.0(0.8,1.4)$ & $1.1(0.8,1.5)$ & $0.9(0.7,1.3)$ & $1.0(0.7,1.4)$ & $1.2(0.9,1.5)$ & 0.451 \\
\hline Model 2 & $0.9(0.7,1.2)$ & $1.1(0.8,1.4)$ & Reference & $1.0(0.7,1.3)$ & $1.2(0.9,1.6)$ & $1.0(0.7,1.3)$ & $1.1(0.8,1.4)$ & $1.1(0.8,1.5)$ & $0.9(0.7,1.3)$ & $1.0(0.7,1.5)$ & $1.2(0.9,1.5)$ & 0.489 \\
\hline Model 3 & $1.0(0.7,1.3)$ & $1.1(0.8,1.4)$ & Reference & $1.0(0.7,1.2)$ & $1.2(0.9,1.5)$ & $0.9(0.7,1.2)$ & $1.0(0.7,1.3)$ & $1.1(0.8,1.4)$ & $0.9(0.6,1.2)$ & $0.9(0.6,1.4)$ & $1.0(0.8,1.4)$ & 0.433 \\
\hline Model 4 & $1.0(0.7,1.3)$ & $1.1(0.8,1.5)$ & Reference & $1.0(0.7,1.3)$ & $1.2(0.9,1.6)$ & $0.9(0.7,1.2)$ & $1.0(0.8,1.4)$ & $1.1(0.8,1.4)$ & $0.9(0.6,1.2)$ & $1.0(0.7,1.5)$ & $1.0(0.8,1.4)$ & 0.720 \\
\hline \multicolumn{13}{|c|}{ Hypercholesterolemia } \\
\hline Model $1^{2}$ & $1.2(0.8,2.0)$ & $1.5(1.0,2.5) *$ & Reference & $1.4(0.9,2.2)$ & $1.7(1.1,2.7) *$ & $1.8(1.2,2.9) *$ & $1.3(0.8,2.2)$ & $1.1(0.6,1.9)$ & $1.4(0.8,2.5)$ & $1.8(1.0,3.3) *$ & $2.2(1.5,3.4) *$ & 0.066 \\
\hline Model 2 & $1.2(0.8,2.0)$ & $1.5(1.0,2.5)$ * & Reference & $1.4(0.9,2.2)$ & $1.7(1.1,2.7) *$ & $1.8(1.2,2.9) *$ & $1.4(0.8,2.3)$ & $1.1(0.7,1.9)$ & $1.4(0.8,2.5)$ & $1.8(1.0,3.3) *$ & $2.2(1.5,3.4)$ * & 0.072 \\
\hline Model 3 & $1.3(0.8,2.2)$ & $1.5(1.0,2.5)$ * & Reference & $1.3(0.8,2.2)$ & $1.6(1.0,2.6)^{*}$ & $1.7(1.1,2.8)^{*}$ & $1.3(0.8,2.1)$ & $1.1(0.6,1.8)$ & $1.3(0.7,2.3)$ & $1.7(0.9,3.1)$ & $2.1(1.3,3.3)$ * & 0.054 \\
\hline Model 4 & $1.3(0.8,2.1)$ & $1.6(1.0,2.5)^{*}$ & Reference & $1.3(0.8,2.2)$ & $1.6(1.0,2.6)^{*}$ & $1.7(1.1,2.8)^{*}$ & $1.3(0.8,2.2)$ & $1.0(0.6,1.8)$ & $1.3(0.7,2.3)$ & $1.7(0.9,3.2)$ & $2.0(1.3,3.3)$ * & 0.057 \\
\hline \multicolumn{13}{|c|}{ Hypertriglyceridemia } \\
\hline Model 12 & $1.0(0.7,1.5)$ & $1.3(0.9,1.9)$ & Reference & $1.2(0.8,1.7)$ & $1.4(0.9,2.0)$ & $0.9(0.6,1.3)$ & $1.1(0.7,1.6)$ & $1.0(0.6,1.4)$ & $0.8(0.5,1.2)$ & $0.6(0.3,1.1)$ & $1.2(0.9,1.7)$ & 0.635 \\
\hline Model 2 & $1.0(0.7,1.5)$ & $1.3(0.9,1.9)$ & Reference & $1.2(0.8,1.7)$ & $1.4(1.0,1.9)$ & $0.9(0.6,1.3)$ & $1.1(0.7,1.6)$ & $1.0(0.7,1.5)$ & $0.7(0.5,1.2)$ & $0.6(0.3,1.1)$ & $1.2(0.9,1.7)$ & 0.597 \\
\hline Model 3 & $1.1(0.7,1.6)$ & $1.3(0.9,1.9)$ & Reference & $1.1(0.8,1.6)$ & $1.3(0.9,1.8)$ & $0.9(0.6,1.3)$ & $1.0(0.7,1.4)$ & $0.9(0.6,1.4)$ & $0.7(0.4,1.1)$ & $0.5(0.3,1.0)$ & $1.0(0.7,1.5)$ & 0.090 \\
\hline Model 4 & $1.1(0.7,1.6)$ & $1.4(0.9,2.0)$ & Reference & $1.1(0.8,1.7)$ & $1.3(0.9,1.9)$ & $0.9(0.6,1.3)$ & $1.0(0.7,1.5)$ & $0.9(0.6,1.4)$ & $0.7(0.4,1.1)$ & $0.6(0.3,1.1)$ & $1.0(0.7,1.5)$ & 0.080 \\
\hline \multicolumn{13}{|c|}{ HDL-hypocholesterolemia } \\
\hline Model 12 & $0.8(0.6,1.1)$ & $0.9(0.7,1.3)$ & Referer & $0.8(0.6,1.1)$ & $0.9(0.7,1.3)$ & $0.7(0.5,1.0)$ & $0.9(0.7,1.3)$ & $0.9(0.6,1.2)$ & $0.7(0.5,1.1)$ & $0.8(0.5,1.2)$ & $0.7(0.5,1.0)$ & 0.824 \\
\hline Model 2 & $0.8(0.6,1.1)$ & $0.9(0.7,1.3)$ & Reference & $0.8(0.6,1.1)$ & $0.9(0.7,1.2)$ & $0.7(0.5,1.0)$ & $09\left(071_{1}^{1}\right)$ & $0.9(0.6,1.2)$ & $0.7(0.5,1.1)$ & $0.8(0.5,1.2)$ & $0.7(0.5,1.0)$ & 0.827 \\
\hline Model 3 & $0.9(0.6,1.2)$ & $1.0(0.7,1.3)$ & Reference & $0.8(0.6,1.1)$ & $0.9(0.7,1.2)$ & $0.7(0.5,1.0)$ & $0.9(0.6,1.2)$ & $0.8(0.6,1.2)$ & $0.7(0.5,1.0)$ & $0.7(0.5,1.2)$ & $0.7(0.5,1.0)$ & 0.795 \\
\hline Model 4 & $0.9(0.6,1.2)$ & $1.0(0.7,1.3)$ & Reference & $0.8(0.6,1.1)$ & $0.9(0.7,1.2)$ & $0.7(0.5,1.0)$ & $0.9(0.7,1.3)$ & $0.8(0.6,1.2)$ & $0.7(0.5,1.1)$ & $0.8(0.5,1.3)$ & $0.7(0.5,1.0)$ & 0.514 \\
\hline \multicolumn{13}{|c|}{ LDL-hypercholesterolemia } \\
\hline Model $1^{2}$ & $1.1(0.7,1.6)$ & $1.1(0.7,1.7)$ & Reference & $1.3(0.9,2.0)$ & $1.6(1.1,2.4)^{*}$ & $1.5(1,2.3 .0) *$ & $1.4(0.9,2.1)$ & $1.5(0.9,2.3)$ & $1.6(1.0,2.6)^{*}$ & $1.2(0.7,2.2)$ & $2.2(1.5,3.2) *$ & 0.149 \\
\hline Model 2 & $1.1(0.7,1.6)$ & $1.1(0.7,1.7)$ & Reference & $1.3(0.9,2.0)$ & $1.6(1.1,2.4)$ * & $1.5(1,2.3 .0) *$ & $1.4(0.9,2.2)$ & $1.5(0.9,2.3)$ & $1.6(1.0,2.6)$ * & $1.2(0.7,2.2)$ & $2.2(1.5,3.2)$ * & 0.160 \\
\hline Model 3 & $1.1(0.7,1.7)$ & $1.1(0.7,1.7)$ & Reference & $1.3(0.9,1.9)$ & $1.5(1.0,2.3)^{*}$ & $1.5(1.0,2.2) *$ & $1.3(0.9,2.1)$ & $1.4(0.9,2.1)$ & $1.5(0.9,2.4)$ & $1.1(0.6,2.1)$ & $2.0(1.3,3.0) *$ & 0.132 \\
\hline Model 4 & $1.1(0.7,1.7)$ & $1.1(0.7,1.8)$ & Reference & $1.3(0.9,2.0)$ & $1.6(1.0,2.3)$ * & $1.5(1.0,2.2)$ * & $1.4(0.9,2.1)$ & $1.4(0.9,2.2)$ & $1.5(0.9,2.5)$ & $1.2(0.6,2.1)$ & $2.0(1.3,3.0)$ * & 0.177 \\
\hline
\end{tabular}

$* p<0.05$. ${ }^{1}$ : We calculated the $p$-trend by assigning median values to levels of dietary cholesterol intake and entered this variable as a continuous term in the regression models. 2: We adjusted multivariable logistic regression models for gender, age (18-44 years, $45-60$ years), educational level ( $\leq$ primary school, secondary school, $\geq$ high school), yearly income (tertiles), geographic region (Model 1), current smoking and drinking statuses (yes, no), physical activity (continuous; Model 2), intake of energy (continuous), carbohydrate (continuous), fat (continuous), protein (continuous), fiber (continuous), vitamin C (continuous), vitamin E (continuous), niacin (continuous), selenium (continuous; Model 3), hypertension (yes, no), and BMI (continuous; Model 4). 


\section{Discussion}

Using CHNS 2015, the present study examined the prevalence of dyslipidemia and its subtypes and the impact of dietary cholesterol intake levels on them. It was found that an estimated $37.5 \%$ of Chinese adults aged 18-59 years in our study had at least one type of dyslipidemia, which was higher than $34.0 \%$ of Chinese adults aged $\geq 18$ years from a representative research of the China National Survey of Chronic Kidney Disease [23] and 16.8\% of Korean adults aged 40-64 years from a cross-sectional analysis of the National Health Insurance Service-National Sample Cohort database [24]. Furthermore, our study showed that approximately $10 \%$ of adults have hypercholesterolemia, $15.2 \%$ hypertriglyceridemia, 12.7\% LDL-hypercholesterolemia, and more than 20\% HDL-hypocholesterolemia. Then, the prevalence of hypercholesterolemia, LDL-hypercholesterolemia, and HDL-hypocholesterolemia increased about $1 \%, 2 \%$, and $6 \%$, respectively from CHNS 2009 to the CHNS 2015, whereas the prevalence of hypertriglyceridemia decreased $8 \%$ [25].

The refining grouping of dietary cholesterol intake with the top-level at $\geq 500 \mathrm{mg} /$ day makes it possible to fully explore its dose-response association with dyslipidemia and its subtypes. Previous studies suggested that adults with lower total cholesterol in the blood $(\leq 160 \mathrm{mg} / \mathrm{dL})$ have a higher mortality rate [26,27], and a large-scale observational study of residents aged 35-89 in Hiroshima and Nagasaki found a significant $64 \%$ lower risk of stroke in people with a median cholesterol intake of $624 \mathrm{mg} /$ day compared to those with a median cholesterol intake of $152 \mathrm{mg} /$ day [28]. Our study found the lowest prevalence of hypercholesterolemia and LDL-hypercholesterolemia at a cholesterol intake level of $100.0-150.0 \mathrm{mg} /$ day, rather than at level of $<100 \mathrm{mg} /$ day.

Further, it was only observed that adults with the top level of dietary cholesterol intake had significantly higher odds of hypercholesterolemia as well as LDL-hypercholesterolemia as compared with those with 100.0-150.0 mg/day. And no significant linear trends between them was observed, which may indicate the potential threshold-effect association between them. One prospective study suggested the cholesterol intake of $\geq 747.0 \mathrm{mg} /$ day associated with increased risk of ischemic heart disease and cancer in Japanese middle-aged men (45-68 years) living in Hawaii [29]. Previous studies, defining the grouping of dietary cholesterol intake as $\geq 300 \mathrm{mg} /$ day and $<300 \mathrm{mg} /$ day, had limited ability to discover the potential association between dietary cholesterol intake and dyslipidemia and may underestimate the effect magnitude $[13,30]$. Some studies choosing the lowest dietary cholesterol intake as the reference group may conceal potential adverse impact on dyslipidemia or subtypes in relation to extremely low intake versus reasonable intake.

Our study has several limitations. First, our study assessed dietary intake by 3 days $24 \mathrm{~h}$ recalls, which may have a relatively limited correction for within-subject variation. It posed the risk of underestimating the dietary intakes, especially for episodically consumed foods as compared with nonconsecutive $24 \mathrm{~h}$ recalls. However, the average intake over three days can offer a relatively valid estimate of usual diet, as has been shown in earlier research using the CHNS [31]. Second, although a number of potential confounding factors were adjusted, we still cannot avoid the possibility of residual confounding. Third, there was no information on the use of cholesterol-lowering medication, which may underestimate the prevalence odds of dyslipidemia in relation to the dietary cholesterol intakes. Fourth, our study only identified apoplexy in general as exclusion criteria given the lack of information to distinguish cerebral infarction from cerebral bleeding. In addition, the cross-sectional nature of our study does not allow us to evaluate causal associations between dietary cholesterol and dyslipidemia risks. Prospective studies with large sample sizes would be required to further investigate this association.

\section{Conclusions}

In conclusion, this study indicates high prevalence of dyslipidemia dominating hypertriglyceridemia and HDL-hypocholesterolemia in Chinese adults and a dietary cholesterol intake level of 100-150 mg/day in relation to the lowest prevalence of hypercholesterolemia and LDL-hypercholesterolemia. A dietary cholesterol intake level of $500 \mathrm{mg} /$ day and above may be a 
threshold point for high odds of hypercholesterolemia and LDL-hypercholesterolemia. Keeping daily dietary cholesterol intake within a reasonable range in a healthy eating pattern should be recommended. Findings from our study have implications for nutrition intervention targeting adults with relatively high levels of dietary cholesterol intake. Whether one limited intake of $500 \mathrm{mg} /$ day should be recommended requires large-scale prospective cohort studies on dietary cholesterol intake-health outcomes.

Author Contributions: Writing—original draft preparation, Q.H.; project administration, B.Z.; methodology, Z.W., L.W., and Q.H.; investigation, H.J., X.J., and F.H.; supervision, H.W.; data analysis, Q.H.; manuscript review, Z.W.

Funding: This research was supported by the National Institute for Nutrition and Health, China Center for Disease Control and Prevention; Carolina Population Center (5 R24 HD050924), the University of North Carolina at Chapel Hill; the National Institutes of Health (R01-HD30880, DK056350, R24 HD050924, R01-HD38700); the Fogarty International Center, National Institutes of Health (5D43TW007709 and 5D43TW009077); and the Ministry of Finance of the People's Republic of China, the China Nutritional Transition Cohort Study (13103110700015005). All funders had no role in the design, analysis, or writing of this article.

Acknowledgments: The present study uses data from the CHNS. We thank all of the participants and staff involved in the surveys.

Conflicts of Interest: The authors declare no conflict of interest.

\section{References}

1. National Cholesterol Education Program (NCEP) Expert Panel on Detection, Evaluation, and Treatment of High Blood Cholesterol in Adults (Adult Treatment Panel III). Third Report of the National Cholesterol Education Program (NCEP) Expert Panel on Detection, Evaluation, and Treatment of High Blood Cholesterol in Adults (Adult Treatment Panel III) final report. Circulation 2002, 106, 3143-3421. [CrossRef]

2. Heart Protection Study Collaborative Group. MRC/BHF Heart Protection Study of cholesterol lowering with simvastatin in 20,536 high-risk individuals: A randomised placebo-controlled trial. Lancet 2002, 360, 7-22. [CrossRef]

3. Baigent, C.; Keech, A.; Kearney, P.M.; Blackwell, L.; Buck, G.; Pollicino, C.; Kirby, A.; Sourjina, T.; Peto, R.; Collins, R.; et al. Cholesterol Treatment Trialists: Efficacy and safety of cholesterol-lowering treatment: Prospective meta-analysis of data from 90,056 participants in 14 randomised trials of statins. Lancet 2005, 366, 1267-1278. [PubMed]

4. Grundy, S.M.; Cleeman, J.I.; Merz, C.N.; Brewer, H.B.J.; Clark, L.T.; Hunninghake, D.B.; Pasternak, R.C.; Smith, S.C.J.; Stone, N.J.P. Coordinating Committee of the National Cholesterol Education: Implications of recent clinical trials for the national cholesterol education program adult treatment Panel III Guidelines. J. Am. Coll. Cardiol. 2004, 44, 720-732. [CrossRef]

5. Sun, G.Z.; Li, Z.; Guo, L.; Zhou, Y.; Yang, H.M.; Sun, Y.X. High prevalence of dyslipidemia and associated risk factors among rural Chinese adults. Lipids Health Dis. 2014, 13, 189. [CrossRef] [PubMed]

6. Mente, A.; Dehghan, M.; Rangarajan, S.; McQueen, M.; Dagenais, G.; Wielgosz, A.; Lear, S.; Li, W.; Chen, H.; Yi, S.; et al. Association of dietary nutrients with blood lipids and blood pressure in 18 countries: A cross-sectional analysis from the PURE study. Lancet Diabetes Endocrinol. 2017, 5, 774-787. [CrossRef]

7. Weggemans, R.M.; Zock, P.L.; Katan, M.B. Dietary cholesterol from eggs increases the ratio of total cholesterol to high-density lipoprotein cholesterol in humans: A meta-analysis. Am. J. Clin. Nutr. 2001, 73, 885-891. [CrossRef]

8. McNamara, D.J. The impact of egg limitations on coronary heart disease risk: Do the numbers add up? J. Am. Coll. Nutr. 2000, 19, 540S-548S. [CrossRef]

9. Dawber, T.R.; Nickerson, R.J.; Brand, F.N.; Pool, J. Eggs, serum cholesterol, and coronary heart disease. Am. J. Clin. Nutr. 1982, 36, 617-625. [CrossRef]

10. Soliman, G.A. Dietary Cholesterol and the Lack of Evidence in Cardiovascular Disease. Nutrients 2018, 10, E780. [CrossRef] [PubMed]

11. Hu, Y.W.; Zheng, L.; Wang, Q. Regulation of cholesterol homeostasis by liver X receptors. Clin. Chim. Acta 2010, 411, 617-625. [CrossRef] [PubMed] 
12. Lin, E.C.; Fernandez, M.L.; McNamara, D.J. Dietary fat type and cholesterol quantity interact to affect cholesterol metabolism in guinea pigs. J. Nutr. 1992, 122, 2019-2029. [CrossRef] [PubMed]

13. Su, C.; Jia, X.; Wang, Z.; Wang, H.; Zhang, B. Study on the relationships between dietary cholesterol and serum cholesterol among Chinese adults. Zhonghua Liu Xing Bing Xue Za Zhi 2015, 36, 842-845. (In Chinese) [PubMed]

14. Zhu, Z.; Wu, F.; Lu, Y.; Wang, Z.; Zang, J.; Yu, H.; Guo, C.; Jia, X.; Shen, X.; Ding, G. The Association of Dietary Cholesterol and Fatty Acids with Dyslipidemia in Chinese Metropolitan Men and Women. Nutrients 2018, 10, 961. [CrossRef]

15. Van Horn, L.; Carson, J.A.; Appel, L.J.; Burke, L.E.; Economos, C.; Karmally, W.; Lancaster, K.; Lichtenstein, A.H.; Johnson, R.K.; Thomas, R.J.; et al. Recommended Dietary Pattern to Achieve Adherence to the American Heart Association/American College of Cardiology (AHA/ACC) Guidelines: A Scientific Statement from the American Heart Association. Circulation 2016, 134, e505-e529. [CrossRef]

16. Wang, S.S.; Lay, S.; Yu, H.N.; Shen, S.R. Dietary Guidelines for Chinese Residents (2016): Comments and comparisons. J. Zhejiang Univ. Sci. B 2016, 17, 649-656. [CrossRef]

17. Jiang, H.R.; Wang, Z.H.; Ou, Y.Y.Y.; Jia, X.F.; Huang, F.F.; Zhang, B. Trends in Dietary Cholesterol Intake Among Chinese Adults in Nine Provinces and Related Factors from 1991 to 2011. Acta Nutrimenta Sinica 2016, 38, 222-228.

18. Zhang, B.; Zhai, F.Y.; Du, S.F.; Popkin, B.M. The China Health and Nutrition Survey, 1989-2011. Obes. Rev. 2014, 15 (Suppl. 1), 2-7. [CrossRef]

19. Wang, H.J.; Wang, Z.H.; Zhang, J.G.; Du, W.W.; Su, C.; Zhang, J.; Zhai, F.Y.; Zhang, B. Trends in dietary fiber intake in Chinese aged 45 years and above, 1991-2011. Eur. J. Clin. Nutr. 2014, 68, 619-622. [CrossRef]

20. Joint Committee for Developing Chinese guidelines on Prevention and Treatment of Dyslipidemia in Adults. Chinese guidelines on prevention and treatment of dyslipidemia in adults. Zhonghua Xin Xue Guan Bing Za Zhi 2007, 35, 390-419. (In Chinese)

21. Ainsworth, B.E.; Haskell, W.L.; Whitt, M.C.; Irwin, M.L.; Swartz, A.M.; Strath, S.J.; O’Brien, W.L.; Bassett, J.R.; Schmitz, K.H.; Emplaincourt, P.O.; et al. Compendium of Physical Activities: An update of Activity Codes and MET Intensities. Med. Sci. Sports Exerc. 2000, 32 (Suppl. 9), 498-504. [CrossRef] [PubMed]

22. Song, P.K.; Li, H.; Man, Q.Q.; Jia, S.S.; Li, L.X.; Zhang, J. Trends in determinants of hypercholesterolemia among Chinese adults between 2002 and 2012: Results from the national nutrition survey. Nutrients 2017, 9, 279. [CrossRef] [PubMed]

23. Pan, L.; Yang, Z.; Wu, Y.; Yin, R.X.; Liao, Y.; Wang, J.; Gao, B.; Zhang, L.; China National Survey of Chronic Kidney Disease Working Group. The prevalence, awareness, treatment and control of dyslipidemia among adults in China. Atherosclerosis 2016, 248, 2-9. [CrossRef] [PubMed]

24. Boo, S.; Yoon, Y.J.; Oh, H. Evaluating the prevalence, awareness, and control of hypertension, diabetes, and dyslipidemia in Korea using the NHIS-NSC database: A cross-sectional analysis. Medicine 2018, 9, e13713. [CrossRef] [PubMed]

25. Dai, J.; Min, J.Q.; Yang, Y.J. A study on the epidemic characteristics of dyslipidemia in adults of nine provinces of China. Zhonghua Xin Xue Guan Bing Za Zhi 2018, 46, 114-118. (In Chinese)

26. Cui, R.; Iso, H.; Toyoshima, H.; Date, C.; Yamamoto, A.; Kikuchi, S.; Kondo, T.; Watanabe, Y.; Koizumi, A.; JACC Study Group; et al. Serum total cholesterol levels and risk of mortality from stroke and coronary heart disease in Japanese: The JACC study. Atherosclerosis 2007, 194, 415-420. [CrossRef]

27. Okamura, T.; Kadowaki, T.; Hayakawa, T.; Kita, Y.; Okayama, A.; Ueshima, H.; Nippon Data80 Research Group. What cause of mortality can we predict by cholesterol screening in the Japanese general population? J. Intern. Med. 2003, 253, 169-180. [CrossRef]

28. Sauvaget, C.; Nagano, J.; Hayashi, M.; Yamada, M. Animal protein, animal fat, and cholesterol intakes and risk of cerebral infarction mortality in the adult health study. Stroke 2004, 35, 1531-1537. [CrossRef]

29. McGee, D.; Reed, D.; Stemmerman, G.; Rhoads, G.; Yano, K.; Feinleib, M. The relationship of dietary fat and cholesterol to mortality in 10 years: The Honolulu Heart Program. Int J. Epidemiol 1985, 14, 97-105. [CrossRef] 
30. Wang, Y.; Jia, X.; Su, C.; Du, W.; Wang, Z.; Zhang, B.; Wang, H. Dietary cholesterol intake status and the correlation analysis with serum cholesterol level of the residents aged 60 and above in 15 provinces (autonomous regions and municipality) of China in 2015. Wei Sheng Yan Jiu 2019, 48, 33-40. (In Chinese)

31. Paeratakul, S.; Popkin, B.M.; Kohlmeier, L.; Hertz-Picciotto, I.; Guo, X.; Edwards, L.J. Measurement error in dietary data: Implications for the epidemiologic study of the diet-disease relationship. Eur. J. Clin. Nutr. 1998, 52, 722-727. [CrossRef] [PubMed]

(C) 2019 by the authors. Licensee MDPI, Basel, Switzerland. This article is an open access article distributed under the terms and conditions of the Creative Commons Attribution (CC BY) license (http://creativecommons.org/licenses/by/4.0/). 